\title{
Cerebral protection devices for transcatheter aortic valve replacement
}

\author{
Hector Cubero-Gallego ${ }^{1}$, Isaac Pascual ${ }^{1,2,3}$, José Rozado ${ }^{1}$, Ana Ayesta ${ }^{1}$, Daniel Hernandez-Vaquero ${ }^{1,2}$, \\ Rocio Diaz ${ }^{1,2}$, Alberto Alperi ${ }^{1}$, Pablo Avanzas ${ }^{1,2,3}$, Cesar Moris ${ }^{1,2,3}$ \\ ${ }^{1}$ Área del Corazón, Hospital Universitario Central de Asturias, Oviedo, Spain; ${ }^{2}$ Instituto de Investigación Sanitaria del Principado de Asturias, \\ Oviedo, Spain; ${ }^{3}$ Universidad de Oviedo, Departamento de Medicina, Oviedo, Spain \\ Contributions: (I) Conception and design: H Cubero-Gallego, I Pascual, J Rozado, P Avanzas; (II) Administrative support: D Hernandez-Vaquero, C \\ Moris; (III) Provision of study materials or patients: H Cubero-Gallego, A Alperi, R Diaz, C Moris; (IV) Collection and assembly of data: H Cubero- \\ Gallego, I Pascual, A Ayesta, R Diaz; (V) Data analysis and interpretation: H Cubero-Gallego, P Avanzas, D Hernandez-Vaquero; (VI) Manuscript \\ writing: All authors; (VII) Final approval of manuscript: All authors. \\ Correspondence to: Daniel Hernandez-Vaquero, MD, PhD. Área del Corazón, Hospital Universitario Central de Asturias, Oviedo, Spain. \\ Email: dhvaquero@gmail.com.
}

\begin{abstract}
Aortic stenosis is the most prevalent primary valve disease in developed countries. Its prevalence is increasing due to population aging. Transcatheter aortic valve replacement (TAVR) is a sterling therapy for symptomatic patients with severe aortic stenosis and high or intermediate surgery risk. The number of procedures has increased exponentially expanding to younger and lower risk patients. Despite newgeneration TAVR devices and enhanced operator skills, cerebrovascular events (CVEs) carry on being one of the most severe complications, increasing morbi-mortality. CVE might be under reported because there are few studies with rigorous neurological clinical assessment. Several imaging studies show most of CVE after TAVR has a probable embolic etiology. The risk of CVE ranges from $2.7 \%$ to $5.5 \%$ at 30 days. As TAVR expands to younger and lower risk patients, the prevention of stroke plays an increasingly important role. Cerebral protection devices (CPD) were designed to reduce the risk of CVE during TAVR. This review describes the scientific evidence on CVE after TAVR and summarizes the performance and results of the main CPDs.
\end{abstract}

Keywords: Aortic stenosis; transcatheter aortic valve replacement (TAVR); transcatheter aortic valve implantation; cerebrovascular events (CVEs); cerebral protection devices (CPDs); cerebral embolic protection

Submitted Aug 06, 2019. Accepted for publication Aug 30, 2019.

doi: $10.21037 /$ atm.2019.09.25

View this article at: http://dx.doi.org/10.21037/atm.2019.09.25

\section{Introduction}

Aortic stenosis is the most prevalent primary valve disease in developed countries. Its prevalence is increasing due to population aging $(1,2)$. Transcatheter aortic valve replacement (TAVR) is a sterling therapy for symptomatic patients with severe aortic stenosis and high or intermediate surgery risk (1). The number of procedures has increased exponentially and TAVR is expanding to younger and lower risk patients $(3,4)$.

Despite the development of new-generation TAVR devices and enhanced operator skills, cerebrovascular events (CVE) are one of the most severe complications, increasing morbi-mortality $(5,6)$. CVE might be under reported because there are few available studies that use rigorous neurological clinical assessment (7). Several imaging studies showed that most of CVE after TAVR has a probable embolic etiology (8). Usually, risk of CVE ranges from $2.7 \%$ to $5.5 \%$ at 30 days (9). However, some studies have reported up to $10 \%$ stroke rate after a precise neurological assessment (10).

As TAVR expands to younger and lower risk patients, 
the prevention of stroke plays an increasingly important role (11). Cerebral protection devices (CPD) were designed to reduce the risk of CVE during TAVR. Our objective was to describe the etiology, incidence, risk factors and impact of CVE after TAVR and summarizes current evidence of $\mathrm{CPD}$ in this setting.

\section{CVEs during TAVR}

CVE have been under-diagnosed after TAVR, mainly due to the absence of a standardized definition and classification (9). Definitions according the recommendations of the Valve Academic Research Consortium (12) represent one step forward in standardization:

* Transient ischemic attack (TIA): neurological deficit which solves fast $(<24 \mathrm{~h})$, without evidence of tissue injury in imaging study;

* Stroke: new neurological deficit with a longer duration of $24 \mathrm{~h}$, or $<24 \mathrm{~h}$ associated with cerebral injury in imaging study.

Moreover, CVEs can also be classified in accordance to temporality: acute ( $\leq 24 \mathrm{~h})$, subacute (1-30 days), and late (>30 days) events (8).

The incidence of CVE after TAVR shows a significant variability, ranging from 1 to $11 \%$ (13). Studies based on the severity of CVE showed that disabling stroke presented higher incidence (58\%) compared to non-disabling (26\%) and TIA (16\%) (8). A recent meta-analysis showed a median 30 -day stroke rate of $3.1-3.3 \%$ following TAVR, with a 3.5 -fold increase in mortality at 1 -year $(14,15)$.

Peri-procedural CVE are likely to be derived from embolisms associated with manipulation maneuvers and calcium of the stenotic aortic valve. Van Mieghem et al. (16) showed that embolic debris obtained with a CPD during TAVR are usually small with a median size of $1 \mathrm{~mm}$. Studies based on diffusion-weighted magnetic resonance imaging (DW-MRI) reported that $60-90 \%$ of patients showed new silent brain lesions after TAVR, regardless of vascular access (17-19). These lesions were multiple and diffuse suggesting an embolic origin. Studies with transcranial doppler reported high intensity signals (HITS) in the middle cerebral artery (MCA) especially during valve positioning and deployment (20).

Known predictors of CVE after TAVR are the severe calcification of the valve, manipulation maneuvers and mechanical procedural factors (5). Porcelain aorta is associated with enhanced cardiovascular risk but surprisingly, unlike surgical aortic valve replacement, it does not increase the incidence of CVE.

Finally, CVE after TAVR is an independent predictor of morbi-mortality during the follow-up and it has an important impact on life quality, impairing cognitive function and daily abilities $(8,9)$. A classical meta-analysis showed a 3.5 -fold higher 30-day mortality after stroke (21); while a recent meta-analysis, including more than 29,000 patients, reported a 6-fold higher mortality (22).

\section{Neurological protection devices}

Considering that the majority of CVE after TAVR has an embolic etiology, the strategy to reduce CVE is focused on the temporary implantation of mechanical barriers such as cerebral embolic protection devices (CPD) (8). These devices are designed to cover the ostium of the supra-aortic branches in the aortic arch. They are characterized by a low-profile allowing the implantation by the radial artery, filter capabilities and stability during the procedure (9). Despite all this, atherosclerotic plaques close to the ostia of the supra-aortic vessels hinders the correct positioning and stabilization of the device so its use can be challenging (8). Rigorous and careful study of the CT is the key factor in strategic planning.

The CPD can be classified in filters or deflectors. The main characteristics of the CPD are summarized in Table 1 . Filter devices can retain embolic material; while deflector devices reject the debris towards the descending aorta (23). Actually, there are five types of CPD tested during TAVR (23).

Deflector-type systems: Embrella (Edwards Lifesciences, Irvine, CA) and TriGuard (Keystone Heart Ltd, Caesarea, Israel). Filter-type systems: Sentinel (Claret Medical Inc., Santa Rosa, CA), Embol-X (Edwards Lifesciences, Irvine, CA) and Wiron (Allium Medical, Inc., Caesarea, Israel).

\section{Claret sentinel device}

Sentinel Cerebral Protection System (Claret Medical, Inc.; Santa Rosa, California, United States) is a dual system filter basket within a 6 Fr delivery catheter placed percutaneously usually from the right radial over a 0.014 -inch guidewire (Figure 1). It consists of a proximal filter (diameter of 9-15 $\mathrm{mm}$ ) delivered in the brachiocephalic artery and a distal filter (diameter of $6.5-10 \mathrm{~mm}$ ) delivered in the left common carotid artery (Figure 2). Using an articulating sheath, the device can be accommodated into the aortic arch's anatomy (Figure 3) (24). Sentinel is the most widely used CPD; it is positioned before TAVR and is withdrawn 
Table 1 Cerebral protection devices and general characteristics

\begin{tabular}{|c|c|c|c|c|c|c|}
\hline Device & Manufacturer & Access & $\begin{array}{l}\text { Delivery } \\
\text { system }\end{array}$ & Deployment & Design & $\begin{array}{l}\text { Protected cerebral } \\
\text { territories }\end{array}$ \\
\hline Claret-Sentinel & $\begin{array}{l}\text { Claret Medical, Inc., Santa } \\
\text { Rosa, California, United States }\end{array}$ & $\begin{array}{l}\text { Radial/brachial } \\
\text { artery }\end{array}$ & $6 \mathrm{Fr}$ & $\begin{array}{l}\text { Filter to } \\
\text { brachiocephalic trunk } \\
\text { and other to left } \\
\text { common carotid artery }\end{array}$ & Filter & Partial protection \\
\hline Embol-X & $\begin{array}{l}\text { Edwards Lifesciences, Irvine, } \\
\text { California, United States }\end{array}$ & Direct aortic & $14 \mathrm{Fr}$ & Ascending arch & Filter & Full protection \\
\hline TriGuard & $\begin{array}{l}\text { Keystone Heart Ltd., Herzliya, } \\
\text { Israel }\end{array}$ & Femoral artery & $9 \mathrm{Fr}$ & Aortic arch & Deflector & Full protection \\
\hline
\end{tabular}

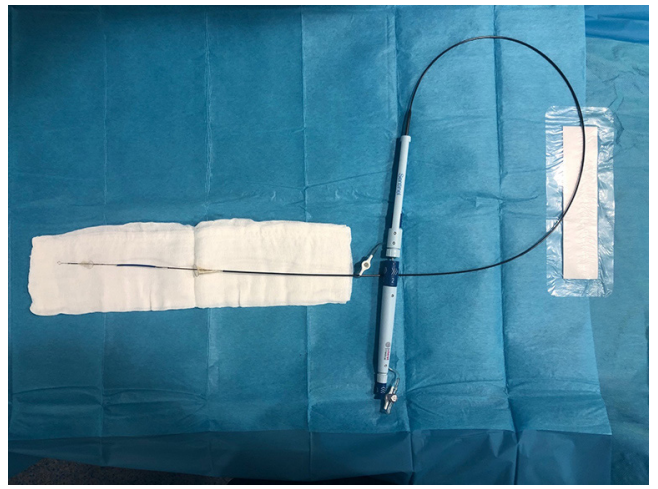

Figure 1 Sentinel cerebral protection system: device.

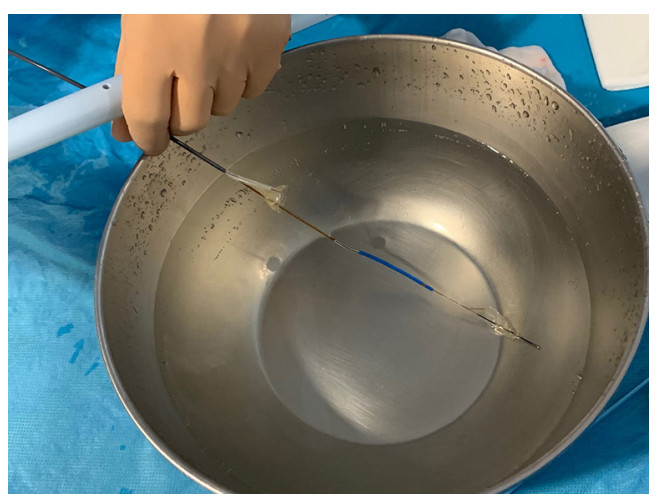

Figure 2 Sentinel cerebral protection system: detail of proximal and distal filters.

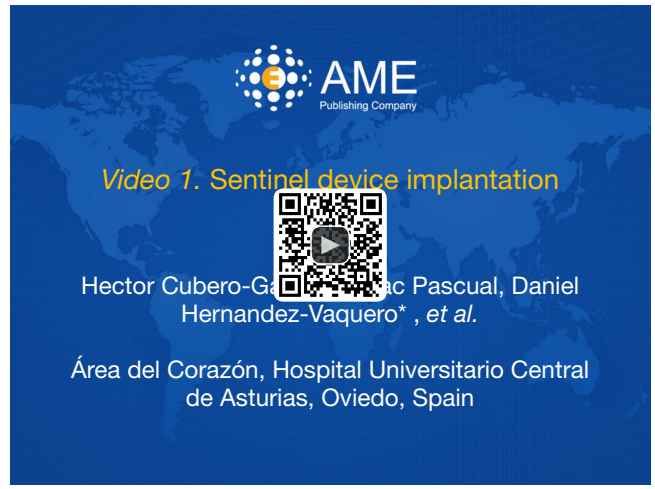

Figure 3 Sentinel device implantation (24).

Available online: http://www.asvide.com/watch/32994

into the catheter and removed after valve implantation $(11,23)$ (Figure 4). Sentinel deployment usually takes less than ten minutes in $91 \%$ of patients undergoing TAVR (9) (Table 1).

Using the Sentinel device, the diameter of the supraaortic vessels must be previously measured by CT because proximal and distal filters are developed to be accommodated within a brachiocephalic artery of 9 to $15 \mathrm{~mm}$ and a common carotid of more than $3 \mathrm{~mm}$. Live $\mathrm{X}$-ray and fusion imaging of CTA-derived 3D anatomical models might be helpful to facilitate the deployment and save contrast dye (25). It is important to point out that 


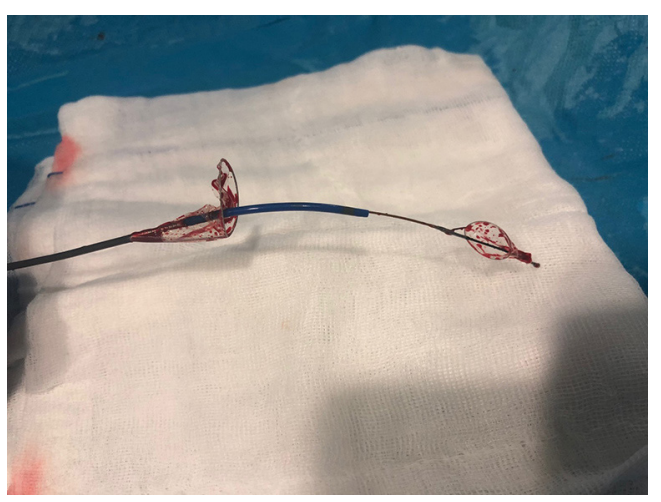

Figure 4 Sentinel cerebral protection system: filter removal.

left vertebral artery, keeps unprotected. This fact could be solved with the use of the Wirion Filter (Allium Medical, Inc.; Caesaria, Israel) deployed into the left vertebral artery (23). Sentinel device has only one available size so complete sealing might not be obtained in all aortic anatomies.

Sentinel is the most studied CPD and has been evaluated in more than one thousand patients. First generation of this device was tested in 2 randomized controlled trials (MISTRAL-C and CLEAN-TAVI) (26,27).

The MISTRAL-C (27) was a multicenter randomized study which included 65 patients. The objective was to compare the number or new cerebral lesions assessed by DW-MRI and the evaluation of neurocognitive function before TAVR and 5 days after TAVR. The filter showed debris in all the patients in the device group. The primary end-point (percentage of patients with new cerebral lesions) was numerically inferior in the device group (73\%vs. 87\%; $\mathrm{P}=0.31$ ) with lower volume of the brain lesions (95 vs. $\left.197 \mathrm{~mm}^{3} ; \mathrm{P}=0.171\right)$. This study showed a significant decrease of patients with more than 10 cerebral lesions (20\%vs. $0 \% ; \mathrm{P}=0.03)$ and less cognitive disability ( $4 \%$ vs. $27 \%$; $\mathrm{P}=0.017$, Table 2).

The CLEAN-TAVI (26), which randomized 100 patients, showed that new cerebral lesions assessed by DW-MRI two days after TAVR. The device group was associated with a decrease of the onset of new brain lesions in protected territories (4 vs. $10 ; \mathrm{P}<0.001)$ and throughout the brain ( 8 vs. 16; $\mathrm{P}=0.002$ ). The volume of the brain lesions was also smaller in the device group (466 vs. $800 \mathrm{~mm}^{3} ; \mathrm{P}=0.02$ ) (Table 2).

Sentinel device was evaluated in 2017 in the largest randomized study to date on CPD. The SENTINEL trial (10) was a multi-center, prospective, single blinded, randomized controlled trial, including 363 patients who underwent TAVR from 19 centers in Germany and US. Patients were randomized 1:1:1 in 3 groups: safety group with the device; and 2 imaging groups which randomly underwent TAVR with or without the CPD. Neurocognitive evaluations and neurologist assessment were meticulously revised before, at 30-day and 90-day follow-up. The CPD was successfully positioned in all the patients, and showed embolic material in $99 \%$ of patients. Primary efficacy end-point (volume of new brain lesions) was comparable between groups (102.8vs. $\left.178 \mathrm{~mm}^{3} ; \mathrm{P}=0.33\right)$. The rate of stroke was numerically inferior in the CPD group although it did not reach a statistically significant difference $(5.6 \%$ vs. $9.1 \%, \mathrm{P}=0.25)$. The results suggest that Sentinel may be safely used and retained debris in most of patients, but without a significant decrease in the onset of new cerebral volume lesion assess by MRI; furthermore, it did not lead to better neurocognitive function. Table 2 shows the main scientific evidence on Sentinel device and another CPD.

\section{Wirion embolic protection system}

The Wirion (Allium Medical Inc.; Caesaria, Israel) is an embolic protection system usually used when carotid artery undergoes stenting which decreases the rate of embolic events (28). Placing the CPD into the left vertebral artery in combination with the Sentinel device, can offer a complete cerebral protection in patients who undergo TAVR (23).

The WISE study (Wirion Study Europe) was a multicenter, non-randomized, trial, which included 120 high surgical risk patients who underwent carotid artery stenting. The primary endpoint (a composite of stroke, myocardial infarction (MI) and death at 30 days) was compared with historical controls results, showing numerically inferior mortality ( $0 \%$ vs. $1.7 \%, \mathrm{P}=0.21)$, stroke $(2.5 \%$ vs. $4.6 \%$, $\mathrm{P}=0.18)$, and $\mathrm{MI}(0.8 \%$ vs. $1.5 \%, \mathrm{P}=0.50)$ with procedural and clinical success of $98.3 \%$ and $96.6 \%$, respectively. The authors concluded that the use of the Wirion in patients with high surgical risk was effective and safe and could be associated with a decrease in the number of complications (28).

\section{Embol $X$ device}

Embol-X (Edwards Lifesciences, CA) is a CPD filter system for cardiac surgery. It requires a direct access to the ascending aorta. A new device version was tested in transaortic TAVR approach with full brain coverage (23) (Table 1). 
Table 2 Current evidence of cerebral protection devices and main characteristics

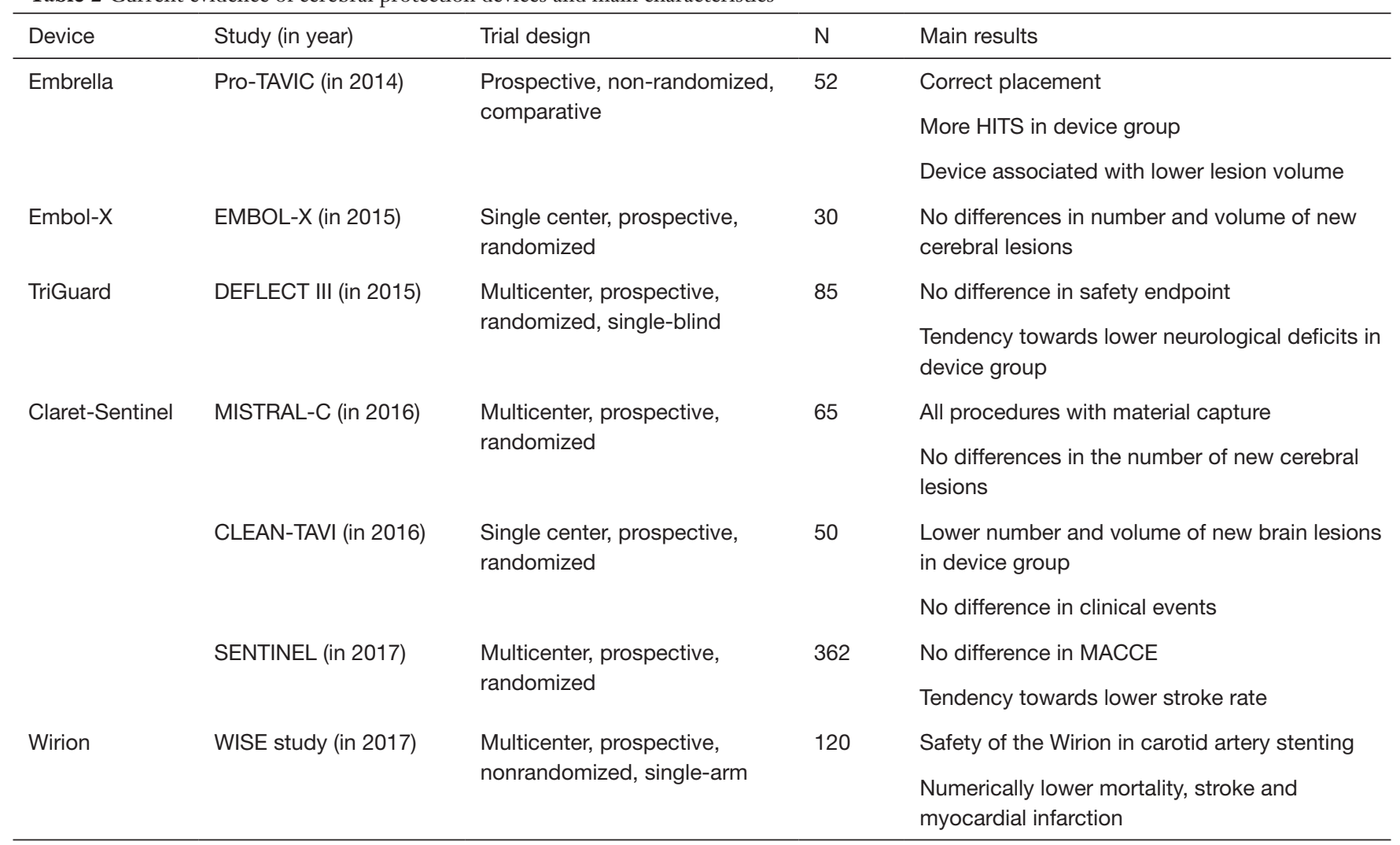

HITS, high intensity signals; MACCE, major adverse cardiac and cerebrovascular events.

Wendt et al. (29) conducted a randomized study which included 30 patients (14 patients with CPD and 16 patients without CPD). The device group showed a nonsignificant decrease in the presence of new cerebral lesions ( $57 \%$ vs. $69 \% ; \mathrm{P}=0.70$ ). The device group showed lower lesion volume in the area supplied by the MCA $(33 \pm 29 v$ s. $76 \pm 67 \mathrm{~mm}^{3}, \mathrm{P}=0.04$ ) (29) (Table 2).

\section{TriGuard device}

The TriGuard device (Keystone Heart Ltd., Herzliya, Israel) is a deflector device developed to reject embolic material during TAVR towards the descending aorta. This deflection device is the only one which offers a full brain coverage $(11,23)$. It is placed through the three brain arteries and is held up by stabilizers (23).

The TriGuard device is advanced through a $9 \mathrm{Fr}$ arterial sheath that is placed into the contralateral femoral artery and is deployed to cover the ostia of the 3 supraaortic trunks (11). Its new generation, the TriGuard 3, incorporates a design by an over-the-wire via a $6 \mathrm{Fr}$ femoral sheath (9) (Table 1). The DEFLECT I (30) and DEFLECT II (31) are studies which showed that the TriGuard devices of first and second generation are safe and effective.

The DEFLECT III (32) is a multicenter, randomized controlled study, including 85 patients, which assessed the efficacy and safety of the TriGuard in TAVR. This study used neurocognitive evaluation and DW-MRI assessment at baseline, before discharge and at 30-day after TAVR. Patients were randomized into 2 groups: CPD group (46 patients) or unprotected group (39 patients). Full coverage was achieved in $89 \%$ of patients. Safety endpoint was not different between both groups occurring in $21.7 \%$ in the TriGuard and $30.8 \%$ in the control group, $\mathrm{P}=0.34$. The use of this device was associated with a non-significant decrease in neurological impairment ( $3.1 \%$ vs. $15.4 \% ; \mathrm{P}=0.16)$, a non-significant decrease of lesions volume and a better efficiency on a retard memory task $(\mathrm{P}=0.028)$ (Table 2).

\section{Embrella device}

The Embrella (Edwards Lifesciences; Irvine, California, 
United States) was a filter developed to deflect embolic material during TAVR (11). This device is inserted by right radial or brachial approach with a 6 Fr sheath (11). The distal end was an oval shaped nitinol frame covered with a porous membrane. The CPD was placed through the greater curvature of the aorta, safeguarding the brachiocephalic and left common carotid artery. Nowadays, this CPD is no longer under development (23) (Table 1).

The PROTAVI-C trial assessed the Embrella device in a study that included 52 patients (42 patients with device and 12 without it) (33). Patients underwent procedural transcranial Doppler and DW-MRI (at baseline, 7 and 30 days). The device did not show prevention of cerebral emboli during TAVR and it presented association with a number of HITs which were much higher than in the control group (632 vs. 279; $\mathrm{P}<0.001$ ). Both groups developed new cerebral lesions assessed by DW-MRI at 1 week after TAVR. However, ischemic lesions were not observed when patients underwent DW-MRI at 30 days. Besides this, it was not associated with cognitive or neurological impairment. The CPD group showed a significant lower volume of lesions ( $\mathrm{P}=0.003$, Table 2).

\section{Conclusions}

Despite great technological advances and the wide experience of the operators, CVE is a severe complication associated with great impact on morbidity, mortality and quality of life. The majority of CVE occurs immediately after TAVR and are due to cerebral embolization. Clinical impact of these "silent" brain lesions must be well defined, particularly for younger patients undergoing TAVR.

CPD were developed as a new therapy to prevent the embolization of debris to the brain. They showed to be safe and effective in reducing cerebral embolisms although current available trials have not been designed to detect differences on clinical impact.

Cerebral protection becomes really relevant since TAVR is expanding to younger and lower risk patients. Further large randomized controlled trials, properly powered with meticulous clinical assessment, are required in order to determine the real role of CPD during TAVR.

\section{Acknowledgments}

Research on cardiovascular diseases is supported by Asturcor Foundation (Oviedo, Spain).

\section{Footnote}

Conflicts of Interest: The authors have no conflicts of interest to declare.

Ethical Statement: The authors are accountable for all aspects of the work in ensuring that questions related to the accuracy or integrity of any part of the work are appropriately investigated and resolved. Written informed consent was obtained from all patients.

\section{References}

1. Baumgartner H, Falk V, Bax JJ, et al. ESC Scientific Document Group 2017 ESC/EACTS Guidelines for the management of valvular heart disease. Eur Heart J 2017;38:2739-91.

2. Osnabrugge RLJ, Mylotte D, Head SJ, et al. Aortic stenosis in the elderly: disease prevalence and number of candidates for transcatheter aortic valve replacement: a meta-analysis and modeling study. J Am Coll Cardiol 2013;62:1002-12.

3. Leon MB, Smith CR, Mack MJ, et al. PARTNER 2 Investigators. Transcatheter or Surgical Aortic-Valve Replacement in Intermediate-Risk Patients. N Engl J Med 2016;374:1609-20.

4. Moat NE. Will TAVR Become the Predominant Method for Treating Severe Aortic Stenosis? N Engl J Med 2016;374:1682-3.

5. Kapadia S, Agarwal S, Miller DC, et al. Insights Into Timing, Risk Factors, and Outcomes of Stroke and Transient Ischemic Attack After Transcatheter Aortic Valve Replacement in the PARTNER Trial (Placement of Aortic Transcatheter Valves). Circ Cardiovasc Interv 2016. doi: 10.1161/CIRCINTERVENTIONS.115.002981.

6. Nombela-Franco L, Webb JG, de Jaegere PP, et al. Timing, predictive factors, and prognostic value of cerebrovascular events in a large cohort of patients undergoing transcatheter aortic valve implantation. Circulation 2012;126:3041-53.

7. Wendler O, Schymik G, Treede H, et al. SOURCE 3 Registry: Design and 30-Day Results of the European Postapproval Registry of the Latest Generation of the SAPIEN 3 Transcatheter Heart Valve. Circulation 2017;135:1123-32.

8. Armijo G, Nombela-Franco L, Tirado-Conte G. Cerebrovascular Events After Transcatheter Aortic Valve Implantation. Front Cardiovasc Med 2018;5:104. 
9. Gasior T, Mangner N, Bijoch J, et al. Cerebral embolic protection systems for transcatheter aortic valve replacement. J Interv Cardiol 2018;31:891-8.

10. Kapadia SR, Kodali S, Makkar R, et al. SENTINEL Trial Investigators. Protection Against Cerebral Embolism During Transcatheter Aortic Valve Replacement. J Am Coll Cardiol 2017;69:367-77.

11. Demir OM, Iannopollo G, Mangieri A, et al. The Role of Cerebral Embolic Protection Devices During Transcatheter Aortic Valve Replacement. Front Cardiovasc Med 2018;5:150.

12. Kappetein AP, Head SJ, Généreux P, et al. Valve Academic Research Consortium (VARC)-2. Updated standardized endpoint definitions for transcatheter aortic valve implantation: the Valve Academic Research Consortium-2 consensus document (VARC-2). Eur J Cardiothorac Surg 2012;42:S45-60.

13. Auffret V, Regueiro A, Del Trigo M, et al. Predictors of Early Cerebrovascular Events in Patients With Aortic Stenosis Undergoing Transcatheter Aortic Valve Replacement. J Am Coll Cardiol 2016;68:673-84.

14. Tay ELW, Gurvitch R, Wijesinghe N, et al. A high-risk period for cerebrovascular events exists after transcatheter aortic valve implantation. JACC Cardiovasc Interv 2011;4:1290-7.

15. Thourani VH, Kodali S, Makkar RR, et al. Transcatheter aortic valve replacement versus surgical valve replacement in intermediate-risk patients: a propensity score analysis. Lancet 2016;387:2218-25.

16. Van Mieghem NM, Schipper MEI, Ladich E, et al. Histopathology of embolic debris captured during transcatheter aortic valve replacement. Circulation 2013;127:2194-201.

17. Kahlert P, Knipp SC, Schlamann M, et al. Silent and apparent cerebral ischemia after percutaneous transfemoral aortic valve implantation: a diffusion-weighted magnetic resonance imaging study. Circulation 2010;121:870-8.

18. Arnold M, Schulz-Heise S, Achenbach S, et al. Embolic cerebral insults after transapical aortic valve implantation detected by magnetic resonance imaging. JACC Cardiovasc Interv 2010;3:1126-32.

19. Ghanem A, Müller A, Nähle CP, et al. Risk and fate of cerebral embolism after transfemoral aortic valve implantation: a prospective pilot study with diffusionweighted magnetic resonance imaging. J Am Coll Cardiol 2010;55:1427-32.

20. Kahlert P, Al-Rashid F, Döttger P, et al. Cerebral embolization during transcatheter aortic valve implantation: a transcranial Doppler study. Circulation 2012;126:1245-55.

21. Eggebrecht H, Schmermund A, Voigtländer T, et al. Risk of stroke after transcatheter aortic valve implantation (TAVI): a meta-analysis of 10,037 published patients. EuroIntervention 2012;8:129-38.

22. Muralidharan A, Thiagarajan K, Van Ham R, et al. Meta-Analysis of Perioperative Stroke and Mortality in Transcatheter Aortic Valve Implantation. Am J Cardiol 2016;118:1031-45.

23. Nombela-Franco L, Armijo G, Tirado-Conte G. Cerebral embolic protection devices during transcatheter aortic valve implantation: clinical versus silent embolism. J Thorac Dis 2018;10:S3604-13.

24. Cubero-Gallego H, Pascual I, Rozado J, et al. Sentinel device implantation. Asvide 2019;6:309. Available online: http://www.asvide.com/watch/32994

25. Vernikouskaya I, Rottbauer W, Gonska B, et al. Imageguidance for transcatheter aortic valve implantation (TAVI) and cerebral embolic protection. Int J Cardiol 2017;249:90-5.

26. Haussig S, Mangner N, Dwyer MG, et al. Effect of a Cerebral Protection Device on Brain Lesions Following Transcatheter Aortic Valve Implantation in Patients With Severe Aortic Stenosis: The CLEAN-TAVI Randomized Clinical Trial. JAMA 2016;316:592-601.

27. Van Mieghem NM, van Gils L, Ahmad H, et al. Filterbased cerebral embolic protection with transcatheter aortic valve implantation: the randomised MISTRAL-C trial. EuroIntervention 2016;12:499-507.

28. Scheinert D, Reimers B, Cremonesi A, et al. WISE (Wirion Study Europe) Pivotal Trial Investigators. Independent Modular Filter for Embolic Protection in Carotid Stenting. Circ Cardiovasc Interv 2017. doi: 10.1161/ CIRCINTERVENTIONS.116.004244.

29. Wendt D, Kleinbongard P, Knipp S, et al. Intraaortic Protection From Embolization in Patients Undergoing Transaortic Transcatheter Aortic Valve Implantation. Ann Thorac Surg 2015;100:686-91.

30. Baumbach A, Mullen M, Brickman AM, et al. Safety and performance of a novel embolic deflection device in patients undergoing transcatheter aortic valve replacement: results from the DEFLECT I study. EuroIntervention 2015;11:75-84.

31. Samim M, van der Worp B, Agostoni P, et al. TriGuardTM $\mathrm{HDH}$ embolic deflection device for cerebral protection during transcatheter aortic valve replacement. Catheter Cardiovasc Interv 2017;89:470-7. 
32. Lansky AJ, Schofer J, Tchetche D, et al. A prospective randomized evaluation of the TriGuardTM HDH embolic DEFLECTion device during transcatheter aortic valve implantation: results from the DEFLECT III trial. Eur Heart J 2015;36:2070-8.

33. Rodés-Cabau J, Kahlert P, Neumann F-J, et al. Feasibility and exploratory efficacy evaluation of the Embrella Embolic Deflector system for the prevention of cerebral emboli in patients undergoing transcatheter aortic valve replacement: the PROTAVI-C pilot study. JACC Cardiovasc Interv 2014;7:1146-55.

Cite this article as: Cubero-Gallego $\mathrm{H}$, Pascual I, Rozado J, Ayesta A, Hernandez-Vaquero D, Diaz R, Alperi A, Avanzas P, Moris C. Cerebral protection devices for transcatheter aortic valve replacement. Ann Transl Med 2019;7(20):584. doi: 10.21037/atm.2019.09.25 